\title{
From Software-Defined Vehicles to Self-Driving Vehicles: A Report on CPSS- Based Parallel Driving
}

\section{Shuangshuang Han}

The State Key Laboratory for Management and Control of Complex Systems, Institute of Automation, Chinese Academy of Sciences, Beijing 100190, China, the Qingdao Academy of Intelligent Industries, Shandong 266000, China, and also with Qingdao Huituo Intelligent

Machine Company, Qingdao, China. E-mail: shuangshuang.han@ia.ac.cn

\section{Dongpu Cao}

The Center for Automotive Engineering, Cranfield University, Cranfield MK43 OAL, U.K., and the Qingdao Academy of Intelligent Industries, Shandong 266000, China.

\section{E-mail:dongp_ca@yahoo.com}

\section{Li Li}

Department of Automation, Tsinghua University, Beijing, China 100084 and also with Jiangsu Province Collaborative Innovation Center of Modern Urban Traffic Technologies, SiPaiLou \#2, Nanjing, China, 210096. E-mail: Ii-li@tsinghua.edu.cn

\section{Lingxi Li}

The Department of Electrical and Computer Engineering, Indiana University-Purdue University at Indianapolis, IN 46202 USA, also with the University of Chinese Academy of Sciences, Beijing 100049, China, and with the Qingdao Academy of Intelligent Industries, Shandong 266000, China.E-mail: II7@iupui.edu

\section{Shengbo Eben Li}

The State Key Laboratory of Automotive Safety and Energy, Department of Automotive

Engineering, Tsinghua University, Beijing, 100084, China. E-mail: lisb04@gmail.com

\section{Nan-Ning Zheng}

Institute of Artificial Intelligence and Robotics, Xi'an Jiaotong University, Xi'an 710049, China, and National Engineering Laboratory of Visual Information Processing Applications, Xi'an Jiaotong University, Xi'an 710049, China. E-mail: nnzheng@mail.xjtu.edu.cn

\section{Fei-Yue Wang}

The State Key Laboratory for Management and Control of Complex Systems, Institute of Automation, Chinese Academy of Sciences, Beijing 100190, China, and also with the Research Center for Military Computational Experiments and Parallel Systems Technology, National University of Defense Technology, Changsha 410073, China. E-mail: feiyue.wang@ia.ac.cn 


\begin{abstract}
On June 11th, 2017, the 28th IEEE Intelligent Vehicles Symposium (IV'2017) was held in Redondo Beach, California, USA. As one of the 8 workshops at IV'2017, the cyber-physical-social systems (CPSS)-based parallel driving (WS'08), organized by the State Key Laboratory for Management and Control of Complex Systems (SKL-MCCS), Institute of Automation, Chinese Academy of Sciences, China, Xi'an Jiaotong University, China, Tsinghua University, China, Indiana University-Purdue University Indianapolis, USA, and Cranfield University, U.K, has attracted both researchers and practitioners in intelligent vehicles. About 60-70 participants from various countries had extensive and deep discussions on definition, challenges and alternative solutions for CPSS-based parallel driving, and widely agreed that it is a novel paradigm of cloud-based automated driving technologies. Six speakers shared their ideas, studies, field applications, and vision for future along these emerging directions from software-defined vehicles to selfdriving vehicles.
\end{abstract}

\section{Introduction}

S elf-driving vehicles have been one of the significant applications within the field of intelligent transportation systems (ITS). A variety of research projects have advanced the enabling technologies in environmental perception and vehicle control and have produced experimental implementations to show how automation technologies could be applied to self-driving vehicles. These have led to major demonstrations all over the world, which have attracted intermittent attention. There has been ongoing academic research as well, largely out of sight of the general public. Currently, the integration of more and more sensors, such as camera, radar, GPS, and communication network technologies, opens up a whole new design space for self-driving vehicles. The concept of future self-driving vehicles does not represent only a vision, but a viable reality due to a new class of emerging wireless ad hoc networks for vehicular environment.

Great efforts have been made to improve driving safety by applying advanced and complicated technologies and high-precision equipments, which result in the increasing cost of self-driving vehicles. However, if simple technologies and conventional equipments are used, driving safety would be cut down for cost reduction. Hence, vehicles are no longer isolated mechanical machines used solely for transportation. They are connected through vehicle-tovehicle (V2V) and vehicle-to-infrastructure (V2I) communications, i.e., connected vehicles. Connected vehicles mean applications, services and technologies that connect a vehicle to other vehicles and its surroundings. Currently, wireless communication technologies including WAVE, UMTS, WiMAX, and LTE-V, are subsequently offering a foundation for the development of the next generation of telematics technology. Conventional Vehicular Ad Hoc Networks (VANETs) [1], [2] are evolving into the Internet of Vehicles (IoV) [3], [4]. The former focuses on information transmission among vehicles; while the latter integrates humans and vehicles, that is, $\mathrm{IoV}$ interconnects vehicles and humans within and around vehicles. By using intelligent systems on vehicles and different cyber-physical systems, IoV combines sensors, vehicles and mobile devices to create a global network system. The main target of $\mathrm{IoV}$ is to combine multiple users, vehicles, things and networks to consistently provide the high-quality, controllable, manageable, operational and credible connection.

However, due to the inseparable relationship between self-driving vehicles and pedestrian/passengers, socialbased relationships and mobility aspects of vehicles and pedestrian have been attracted great attention [5]-[9]. It can be well foreseen that in the coming two to four decades (e.g. up to 2050), the road transportation system would be consisting of a mix of connected vehicles with different levels of automation, which necessitates a unified approach for future smart and safe driving. This considerably motivates the development of cyber-physical-social systems [10], [11] (CPSS)-based parallel driving. It would be the best approach to achieve the optimal balance between safety and cost.

As known, the management and control of self-driving systems are commonly static and offline based on mathematical modeling, computation and simulations. However, it is not easy to derive accurate models for complex self-driving systems to test, evaluate and predict the short-term and long-term actions. The artificial societies, computational experiments and parallel execution (ACP) approach was originally proposed for the purpose of modeling, analysis, management and control of complex systems [12]. Along with ACP-based parallel management and control and its wide real-world applications in the past decade [13]-[19], CPSS-based parallel driving has been steadily developed [20], [21]. This is also greatly correlated with the emerging development in connected and 
automated vehicles. This workshop aims to compile the latest research and development advances in CPSS-based parallel driving, and to present and highlight the emerging new technologies in connected and automated vehicles in China [22]-[27].

\section{CPSS-Based Parallel Driving}

\section{A. The Workshop}

The participants of the workshop agreed that CPSS-based parallel driving, as a new paradigm in intelligent vehicles (IV), will emerge as a new and great direction for IV. The study of parallel driving will provide us with more reliable, safe and low-cost vehicles, and comfortable scenarios if we can use the related strategies and techniques effectively and efficiently.

Six lecturers shared their research and studies from various perspectives in this new area (Fig. 1). The first talk is "Parallel Vehicles Based on the ACP Theory: Safe Trips via Self-Driving" as shown in Fig. 2(a) [22]. With the development of intelligent technologies, self-driving vehicles are considered as a promising solution against accident, traffic congestion and pollution problems. Intelligent vehicle techniques have been the research focus all over the world. However, full self-driving vehicles are still far away from its realization and extensive application due to safety

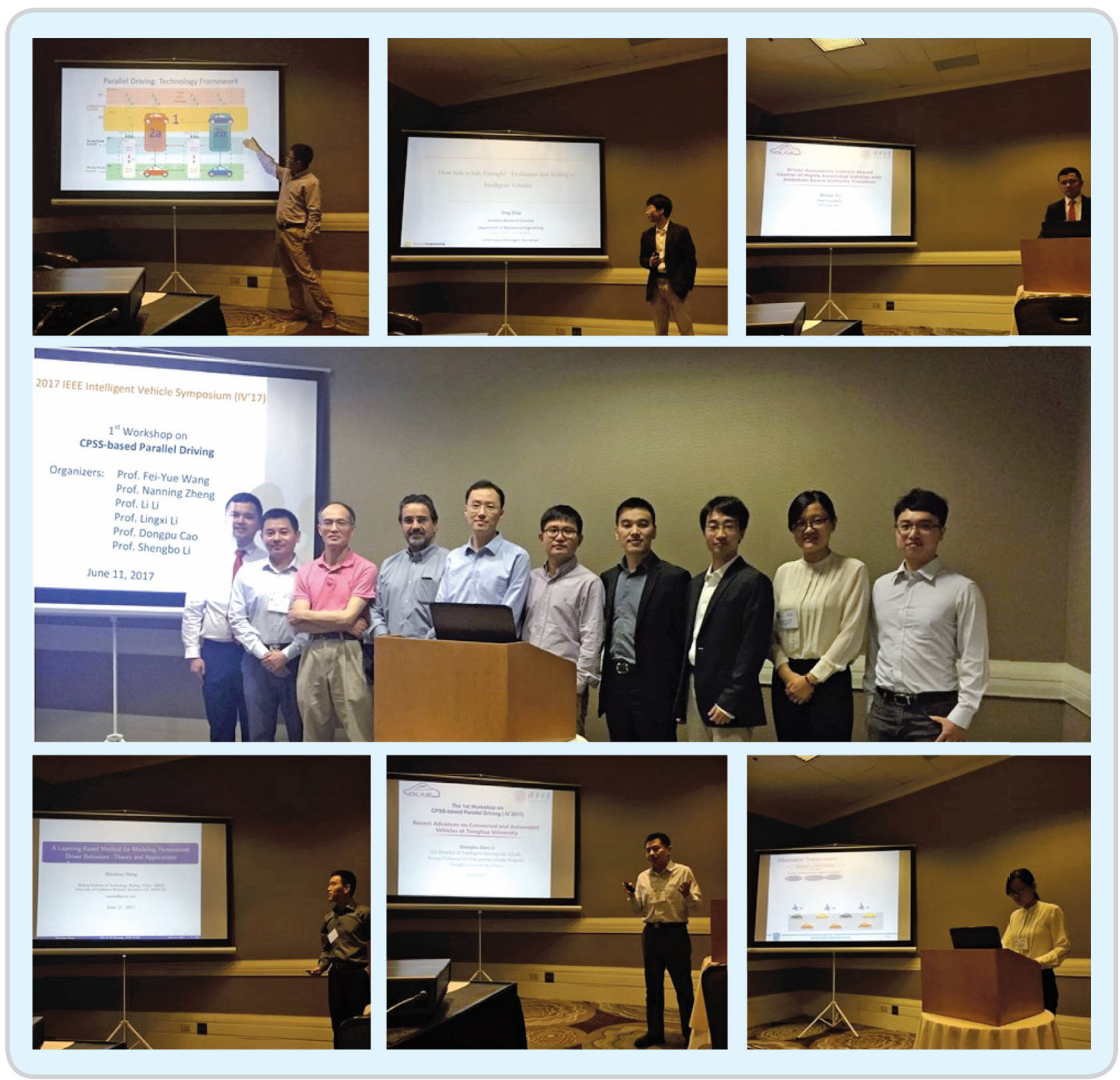

FIG 1 Organizers and lecturers of the workshop. 
requirements and cost considerations. As a novel breakthrough, PArallel VEhicles (PAVE) incorporate the ACP theory, which facilitates real-time interaction and optimization of the actual self-driving vehicles and the artificial ones. As a result, PAVE can maintain intelligent control of the actual self-driving vehicles and achieve the global optimization via software-defined self-driving vehicles, intelligent infrastructure construction, and parallel control center. Besides, PAVE can effectively reduce the cost of high-precision equipment on the actual self-driving vehi- cles via remote processing and intelligent road(side) infrastructure, and also achieve significantly improved safety and reliability via remote control, guidance and planning.

The second talk, from University of Michigan, USA, is titled by "How safe is safe enough?-Evaluation and testing of intelligent vehicles" as shown in Fig. 2(b) [23]. It is necessary to thoroughly evaluate the safety of Automated Vehicles (AVs) before their release and deployment. Current evaluation approach mainly relies on i) testing AVs on public roads or ii) track testing with scenarios defined in a

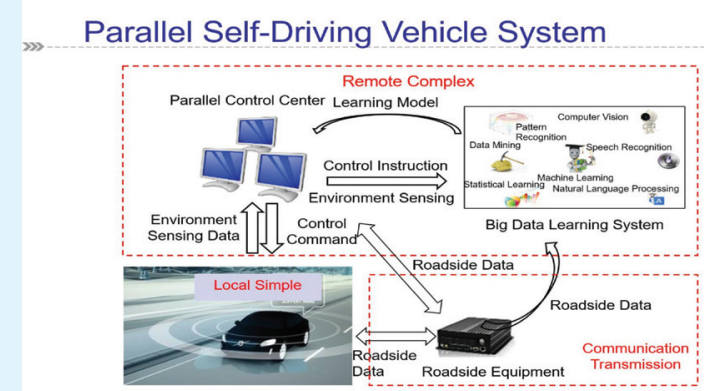

-

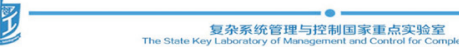

(a)

\section{CDLAB Rearch: Driver Model}

\section{$\square$ Question: How to infer driver's desired $\lambda_{D}$ and $\lambda_{A}$ from his}

Question: How to infer driv
steering angle profile $u_{D}$ ?

$\square$ We need a driver steering model (for indirect shared control)! Previewed

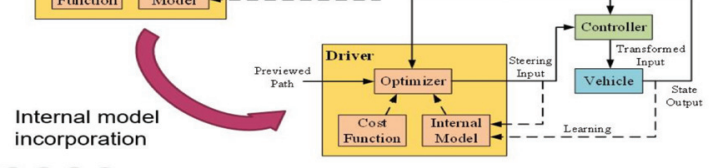

(c)

\section{Research Progress in Decision and Planning}

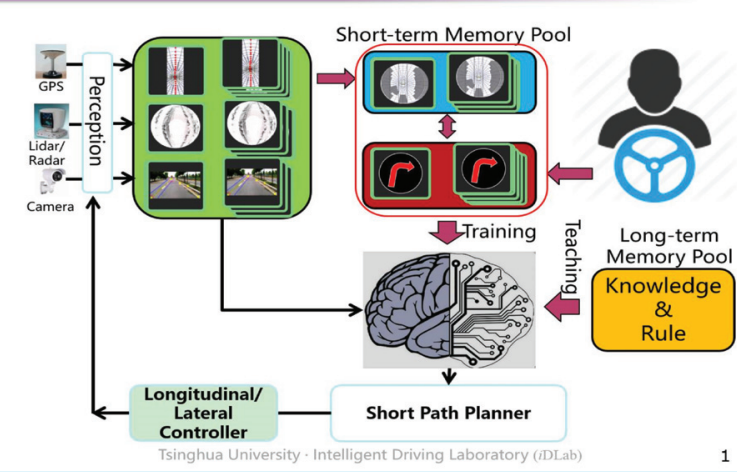

(e)

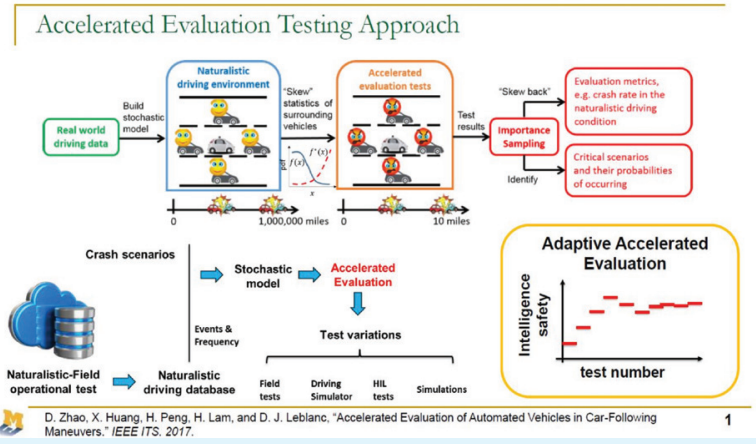

(b)

\section{A Learning-Based Methods for Modeling Personalized Behaviors: Theory and Applications}

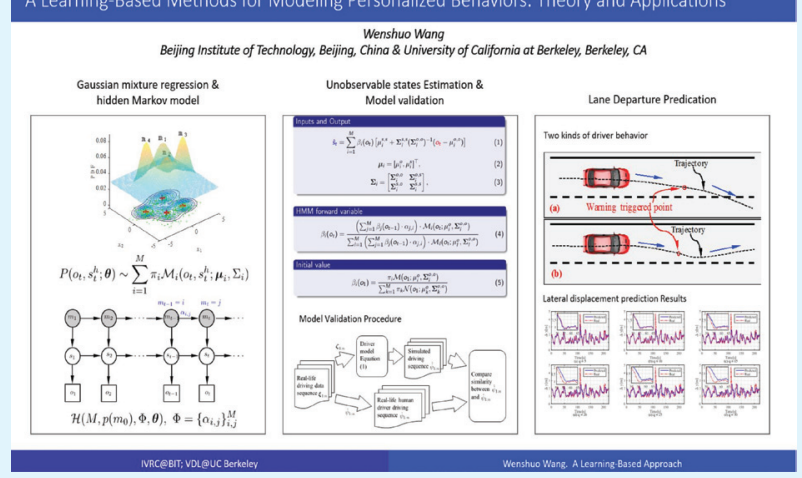

(d)

\section{Parallel Driving}

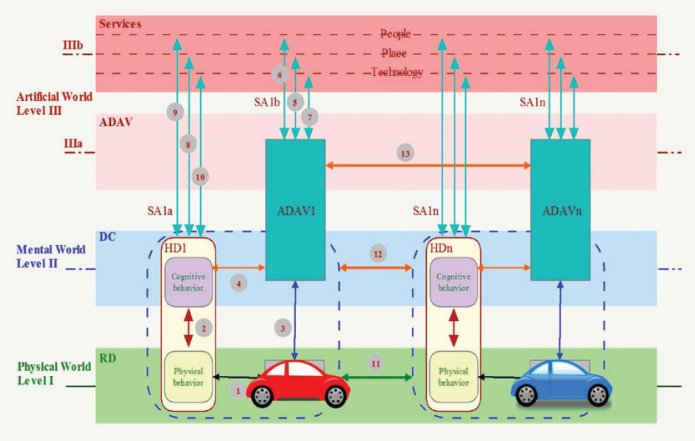

(f)

FIG 2 The main idea of the six talks during the workshop. 
test matrix. These two methods have completely opposite drawbacks: the former takes too much time to execute but is realistic; and the latter can be finished in a short time but has no clear correlation to the safety benefits in the real world.

To avoid the aforementioned problems, they propose the Accelerated Evaluation approach focusing on the lane change and car-following scenarios. The stochastic human-controlled vehicle (HV) motions were modeled based on 1.3 million miles of naturalistic driving data collected by the University of Michigan Safety Pilot Model Deployment Program. The statistics of the HV behaviors were modified to generate more intense interactions between HVs and AVs to accelerate the evaluation procedure. The Importance Sampling theory was used to ensure that the safety benefits of AVs is accurately assessed under accelerated tests. Crash, injury and conflict rates for a simulated $\mathrm{AV}$ are simulated to demonstrate the proposed approach. Results show that the test duration is reduced by a factor of 300 to 100,000 compared with the non-accelerated (natu- ralistic) evaluation. In other words, the proposed techniques have great potential to accelerate the AV evaluation process. Hardware-based validation progress at UofM will also be introduced in the presentation.

The third talk, from Tsinghua University, is titled by "Driver-Automation Indirect Shared Control of Highly Automated Vehicles with Intention-Aware Authority Transition" as shown in Fig. 2(c) [24]. Shared control is an important approach to avoid the driver-out-of-the-loop problems brought by imperfect autonomous driving. Steer-by-wire technology allows the mechanical decoupling between the steering wheel and the road wheels. On steer-by-wire vehicles, the automation can join the control loop by correcting the driver steering input, which forms a new paradigm of shared control. The new framework, under which the driver indirectly controls the vehicle through the automation's input transformation, is called indirect shared control. This paper presents an indirect shared control system, which realizes the dynamic control authority allocation with respect to the driver's authority intention. The simu-

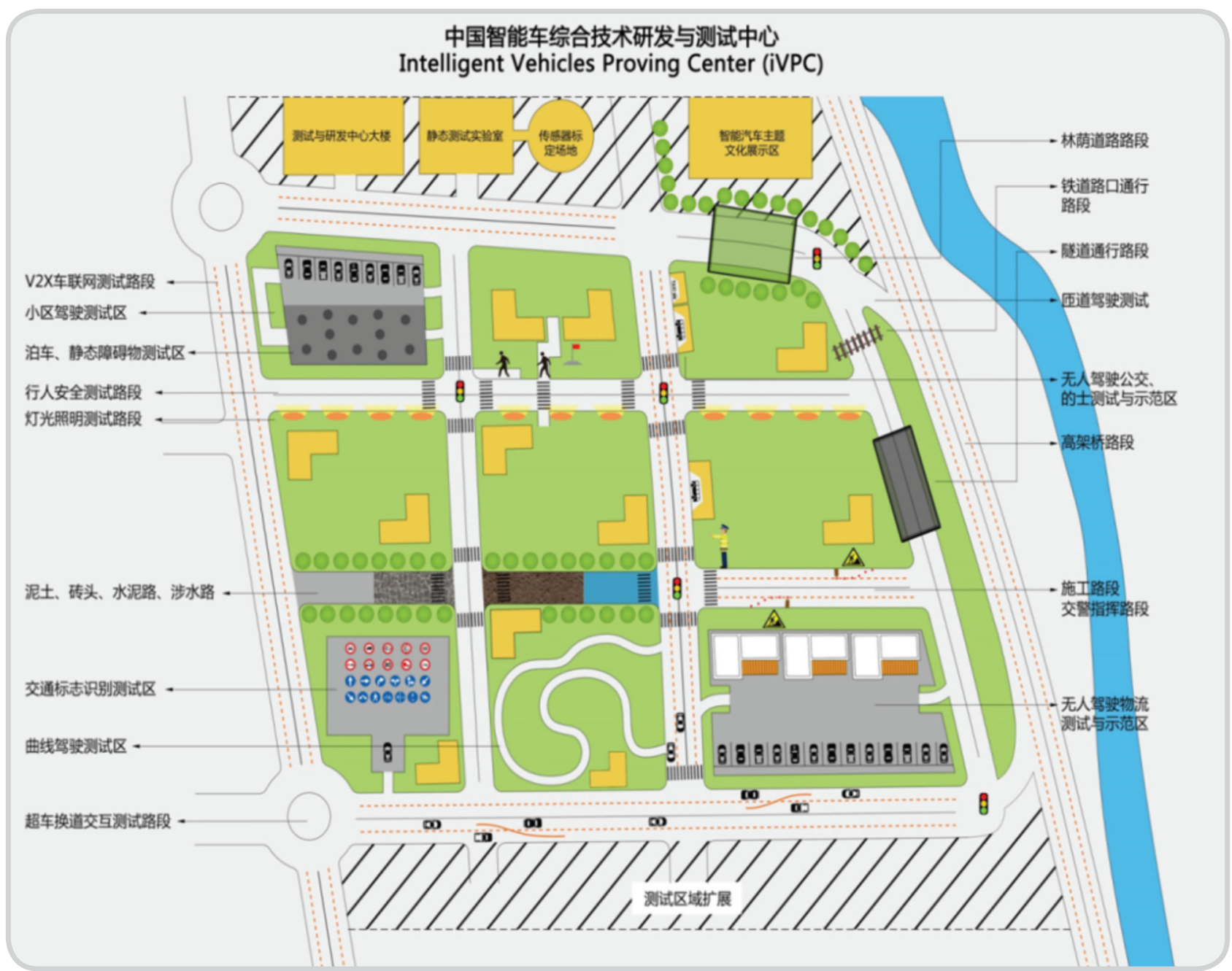

FIG 3 Map of Intelligent Vehicle Proving Center (iVPC). 
lation results demonstrate the effectiveness and benefits of the proposed control authority adaptation method.

The fourth talk, from University of California, Berkeley, USA, presented about "A learning-based method for modeling personalized driver behaviors: theory and application" as shown in Fig. 2(d) [25]. Modeling and understanding driver behaviors bring great challenges for developing ADASs and interactions between human-driven vehicles and autonomous vehicles. In this talk, a learning-based method is shown, where the model structure is established by combing Gaussian mixture model and hidden Markov model. Two case studies in terms of modeling and predicting lateral and longitudinal driver behaviors (i.e., lane departure behavior) are respectively given to show the benefits of our proposed methods.

The fifth talk discussed the recent technical progress in Tsinghua University on transforming driver assistance systems to autonomous vehicles as shown in Fig. 2(e)
[26]. The design of autonomous vehicles is to replace the drivers perception, decision, and manipulation with sensors, controllers and actuators. Any design becomes more challenging when coming to China, especially considering the complexity of mixed traffic, irregularity of road environments, and randomness of driver behaviors. This presentation will talk about new arising challenges and opportunities in autonomous vehicles, especially when facing the complex road environments, driver behaviors, and traffic flow in China. It also talks about main technical progress in Tsinghua University in terms of environmental perception, decision making and dynamical control for autonomous vehicles.

The last talk, from Indiana University-Purdue University Indianapolis, USA, Qingdao Academy of Intelligent Industries, China, and University of Chinese Academy of Sciences, China, presented "Parallel Driving: A Novel Paradigm of Cloud-based Unmanned Driving Technologies” as

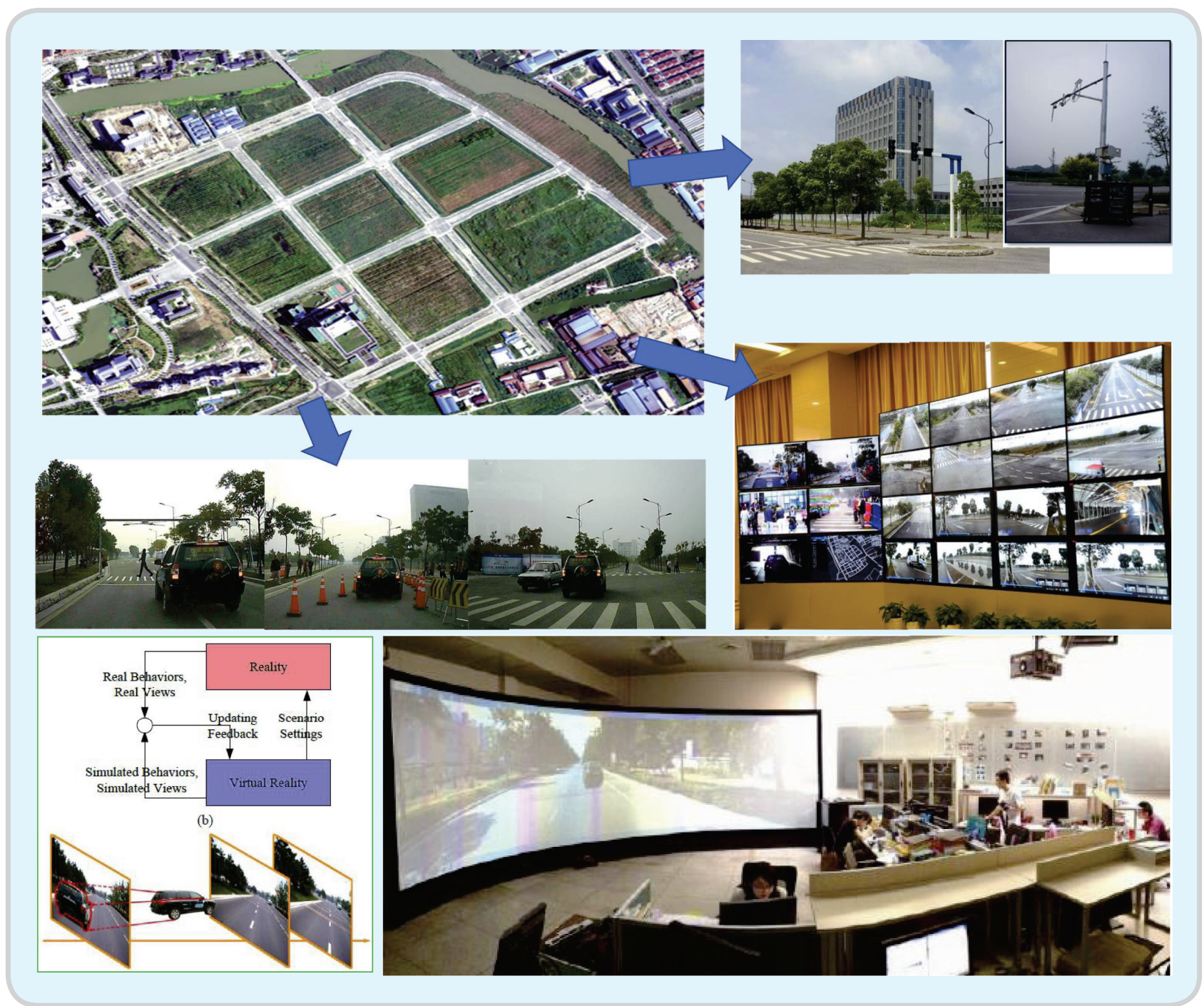

FIG 4 Parallel testing system. 
shown in Fig. 2(f) [27]. Unmanned vehicles and internet of vehicles are two main topics of the current $R \& D$ of intelligent vehicles. How to integrate the technical aspects of these two areas puts forward a big challenge. ACP theorybased parallel driving framework provides a novel solution to enable cloud-based unmanned driving technologies. This talk introduces the latest research progress, technology development, and application examples of parallel driving systems and their subsystems.

\section{B. The Applications}

In 2013 and 2014, two consecutive "intelligent vehicles future challenges (IVFC)" were successfully held by the Changshu Municipal People's Government, Xi'an Jiaotong University, Institute of Automation, Chinese Academy of Sciences, Changan University, and Qingdao Intelligent Industry Technology Research Institute, in High-tech Industrial Development Zone, Changshu City, Jiangsu Province. They then jointly set up Intelligent Vehicle Proving Center (iVPC). The iVPC is positioned as the platform of science and technology innovation platform, public technical service platform and technology trading platform of China Intelligent Automobile Industry. iVPC has owned CNY $150 \mathrm{M}$ investment in 3 years, $20000 \mathrm{~m}^{2}$ static testing area, $350000 \mathrm{~m}^{2}$ proving ground and $2 \mathrm{M} \mathrm{m}^{2}$ expanded testing area (Fig. 3).

After the establishment of iVPC, the seventh and the eighth IVFC were successfully held in 2015 and 2016, respectively. CPSS-based parallel driving testing and evaluation methods (Fig. 4) have been exploited during the eighth IVFC in 2016.

\section{The Perspectives}

Currently, significant R\&D work on CPSS-based parallel driving system has been finished. Now, it is focusing on the R\&D of control\&prediction algorithms, intelligent road(side) system and vehicle/road sensors. Based on the parallel approach and the ACP theory, CPSS-based parallel driving system consists of front-end intelligent vehicles\&infrastructures and background service platform, which are software-defined self-driving vehicle system and remote control system, respectively. The former mainly focus on environment sensing and intelligent vehicle control, etc. The latter is for artificial intelligence, machine learning, and deep learning, etc. By the theoretical foundation and technical support, CPSS-based parallel driving system would be manufactured for different functional modules, such as parallel self-driving logistics vehicles, parallel self-driving bus, parallel self-driving taxi, parallel self-driving subway and so on.

The 2018 IEEE Intelligent Vehicles Symposium (IV'18) will be held in Changshu, Suzhou, China, on June 26-July 1, 2018. Together with IV'18, the Chinese Tenth Intelligent Vehicles Future Challenge (IVFC 2018, June 30-July
1, 2018) will be held at Intelligent Vehicle Proving Center, Changshu, Suzhou, China. Demonstration and Exhibition related the intelligent vehicles are also welcome.

\section{Acknowledgment}

This work was supported partly by the National Natural Science Foundation of China 61533019, 71232006, and 61501461; and the Early Career Development Award of SKLMCCS (Y3S9021F34).

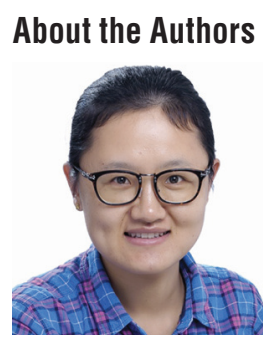

Shuangshuang Han received the Ph.D. degree in the Department of Electrical and Computer Engineering, University of Alberta, Edmonton, AB, Canada, in 2013. Currently, she is an assistant professor with the State Key Laboratory for Management and Control of Complex Systems, Institute of Automation, Chinese Academy of Science. Her research interests include parallel networks, social signal processing, Internet of Vehicles, and performance analysis of parallel network systems.

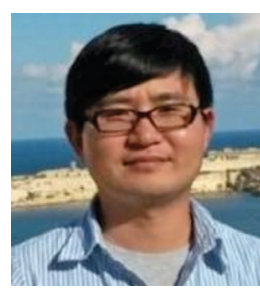

Dongpu Cao received the Ph.D. degree from Concordia University, Canada, in 2008. He is currently an Associate Professor at University of Waterloo, Canada. He received the ASME AVTT2010 Best Paper Award and 2012 SAE Arch T. Colwell Merit Award. His research focuses on vehicle control and intelligence, automated driving and parallel driving, where he has contributed more than 130 publications and 1 US patent.

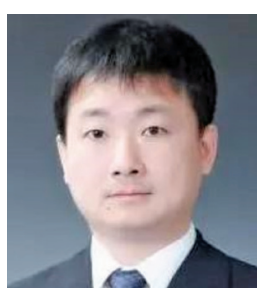

$\boldsymbol{L i} \boldsymbol{L i}$ (S'05-M'06-SM'10-F'17) is currently an associate professor with Department of Automation, Tsinghua University, China. His research interests include complex and networked systems, intelligent control and sensing, intelligent transportation systems and intelligent vehicles. Dr. Li had published over 50 SCI indexed international journal papers and over 50 international conference papers as a first/corresponding author. He serves as an Associate Editor for IEEE Transactions on Intelligent Transportation Systems.

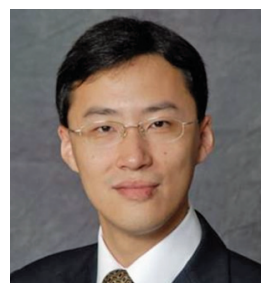

Lingxi $\mathbf{L i}$ received the B.E. degree in automation from Tsinghua University, Beijing, China, in 2000; the M.S. degree in control theory and control engineering from Chinese Academy of Sciences, Beijing, in 2003; and the $\mathrm{Ph} . D$. degree in electrical and computer 
engineering from University of Illinois at Urbana-Champaign, IL, USA, in 2008. Since August 2008, he has been with Indiana University-Purdue University Indianapolis, IN, USA, where he is currently an Associate Professor of electrical and computer engineering. His research interests include modeling, analysis, control, and optimization of complex systems, intelligent transportation systems and intelligent vehicles, discrete event systems, active safety systems, and human factors.

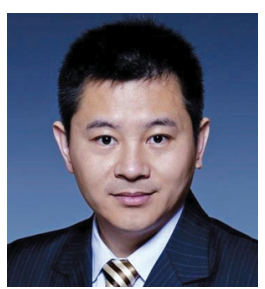

Shengbo Eben $L i$ received the M.S. and Ph.D. degrees from Tsinghua University in 2006 and 2009. He worked as a visiting scholar at Stanford University in 2007, a postdoctoral research fellow in University of Michigan from 2009 to 2011, and a visiting professor in University of California, Berkeley, in 2015. He is the author of more than 80 journal/conference papers, and the co-inventor of more than 20 patents. His active research activities include autonomous vehicle control, driver behaviors and modeling, control topics of battery, optimal control and multi-agent control, etc.

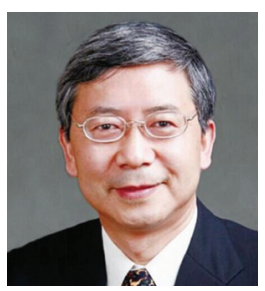

Nan-Ning Zheng (SM'93-F'06) graduated from the Department of Electrical Engineering, Xi'an Jiaotong University, Xi'an, China, in 1975, and received the M.S. degree in information and control engineering from Xi'an Jiaotong University in 1981, and the Ph.D. degree in electrical engineering from Keio University, Yokohama, Japan, in 1985. He joined Xi'an Jiaotong University in 1975, and he is currently a Professor and the Director of the Institute of Artificial Intelligence and Robotics. Prof. Zheng became a member of the Chinese Academy of Engineering in 1999, and he is the Chinese Representative on the Governing Board of the International Association for Pattern Recognition. He also serves as the President of Chinese Association of Automation. His research interests include computer vision, pattern recognition and image processing, and hardware implementation of intelligent systems.

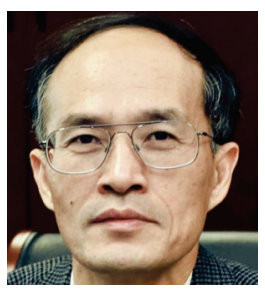

Fei-Yue Wang (S'87-M'89-SM'94-F'03) received his Ph.D. in Computer and Systems Engineering from Rensselaer Polytechnic Institute, Troy, New York in 1990. He joined the University of Arizona in 1990 and became a Professor and Director of the Robotics and Automation Lab (RAL) and Program in Advanced Research for Complex Systems (PARCS). In 1999, he founded the Intelligent Control and Systems Engineering Center at the Institute of Automation, Chinese Academy of Sciences (CAS), Beijing, China, under the support of the Outstanding Overseas Chinese Talents Program from the State Planning Council and "100 Talent Program" from CAS, and in 2002, was appointed as the Director of the Key Lab of Complex Systems and Intelligence Science, CAS. From 2006 to 2010, he was Vice President for Research, Education, and Academic Exchanges at the Institute of Automation, CAS. In 2011, he became the State Specially Appointed Expert and the Director of the State Key Laboratory for Management and Control of Complex Systems. His current research focuses on methods and applications for parallel systems, social computing, parallel intelligence and knowledge automation.

\section{References}

[1] S. Bitam, A. Mellouk, and S. Zeadally, "VANET-cloud: A generic cloud computing model for vehicular Ad Hoc networks," IEEE Wireless Commun., vol. 22, no. 1, pp. 96-102, Feb. 2015.

[2] G. Secinti, B. Canberk, T. Q. Duong, and L. Shu, "Software defined architecture for VANET: A testbed implementation with wireless access management," IEEE Commun. Mag., vol. 55, no. 7, pp. 135-141, 2017.

[3] F. Yang, S. Wang, J. Li, Z. Liu, and Q. Sun, "An overview of Internet of vehicles," China Commun., vol. 11, no. 10, pp. 1-15, Oct. 2014.

[4] J. Cheng, J. Cheng, M. Zhou, F. Liu, S. Gao, and C. Liu, "Routing in Internet of vehicles: A review," IEEE Trans. Intell. Transp. Syst., vol. 16 , no. 5, pp. 2339-2352, Oct. 2015.

[5] A. M. Vegni and V. Loscr, "A survey on vehicular social networks," IEEE Commun. Surveys Tuts., vol. 17, no. 4, pp. 2397-2419, 2015.

[6] M. Lauridsen, L. C. Gimenez, I. Rodriguez, T. B. Sorensen, and P. Mogensen, "From LTE to $5 \mathrm{~g}$ for connected mobility," IEEE Commun. Mag., vol. 55, no. 3, pp. 156-162, Mar. 2017.

[7] Z. Ning, F. Xia, N. Ullah, X. Kong, and X. Hu, "Vehicular social networks: Enabling smart mobility," IEEE Commun. Mag., vol. 55, no. 5, pp. 16-55, May 2017.

[8] S. Correia, A. Boukerche, and R. I. Meneguette, "An architecture for hierarchical software-defined vehicular networks," IEEE Commun. Mag., vol. 55, no. 7, pp. 80-86, 2017.

[9] S. Pagadarai, B. A. Lessard, A. M. Wyglinski, R. Vuyyuru, and O. Altintas, "Vehicular communication: Enhanced networking through dynamic spectrum access," IEEE Veh. Tech. Mag., vol. 8, no. 3, pp. 93-103, Sept. 2013.

[10] F.-Y. Wang, "The emergence of intelligent enterprises: From CPS to CPSS," IEEE Intell. Syst., vol. 25, no. 4, pp. 85-88, July-Aug. 2010.

[11] F.-Y. Wang, X. Wang, L. Li, and L. Li, "Steps toward parallel intelligence," IEEE/CAA J. Autom. Sin., vol. 3, no. 4, pp. 345-348, Oct. 2016.

[12] F.-Y. Wang, "Parallel system methods for management and control of complex systems," Control Decis., vol. 19, no. 5, pp. 485-489, 2004.

[13] F.-Y. Wang, "Parallel control and management for intelligent transportation systems: Concepts, architectures, and applications," IEEE Trans. Intell. Transp. Syst., vol. 11, no. 3, pp. 630-638, Sept. 2010.

[14] F.-Y. Wang, "Agent-based control for networked traffic management systems," IEEE Intell. Syst., vol. 20, no. 5, pp. 92-96, Sept. 2005.

[15] F.-Y. Wang, "Toward a revolution in transportation operations: AI for complex systems," IEEE Intell. Syst., vol. 23, no. 6, pp. 8-13, 2008.

[16] F.-Y. Wang, L. Yang, X. Cheng, S. Han, and J. Yang, "Network softwarization and parallel networks: Beyond software-defined networks," IEEE Netw., vol. 30, no. 4, pp. 60-65, July-Aug. 2016.

[17] F.-Y. Wang, L. Yang, X. Hu, X. Cheng, S. Han, and J. Yang, "Parallel networks and network softwarization: A novel network architecture (in Chinese)," Sci. Sin. Inform., vol. 47, pp. 1-21, 2017.

[18] L. Li, Y. Lin, N. Zheng, and F.-Y. Wang, "Parallel learning: A perspective and a framework," IEEE/CAA J. Autom. Sin., vol. 4, no. 3, pp. 389395, 2017.

[19] L. Li, Y. L. Lin, D. P. Cao, N.-N. Zheng, and F.-Y. Wang, "Parallel learning: A new framework for machine learning," Acta Autom. Sin, vol. 43, no. 1, pp. 1-8, Jan. 2017.

[20] F.-Y. Wang, N.-N. Zheng, D. Cao, and L. Li, "Parallel DRIVING in CPSS: A unified approach from transport automation and vehicle intelligence," submitted for publication.

[21] F.-Y. Wang, "Parallel driving with software vehicular robots for safety and smartness," IEEE Trans. Intell. Transp. Syst., vol. 15, no. 4, pp. 1381-1387, Aug. 2014.

[22] S. Han, F.-Y. Wang, Y. Wang, D. Cao, and L. Li, "Parallel vehicles based on the ACP theory: Safe trips via self-driving," in Proc. 28th IEEE Intell. Vehicles Symp., Los Angeles, CA, 2017, pp. 20-25. 
[23] D. Zhao, "How safe is safe enough? Evaluation and testing of intelligent vehicles," in Proc. 28th IEEE Intell. Vehicles Symp., Los Angeles, CA, 2017.

[24] R. Li, Y. Li, S. Li, E. Burdet, and B. Cheng, "Driver-automation indirec shared control of highly automated vehicles with intention-aware authority transition,” in Proc. 28th IEEE Intelligent Vehicles Symp., Los Angeles, CA, 2017, pp. 26- 32.

[25] W. Wang, "A learning-based method for modeling personalized driver behaviors: theory and application," in Proc. 28th IEEE Intelligent Vehicles Symp., Los Angeles, CA, 2017.
[26] S. E. Li, "Transforming driver assistance systems to autonomous vehicles: Recent technical progress in Tsinghua University," in Proc. 28th IEEE Intelligent Vehicles Symp., Los Angeles, CA, 2017.

[27] L. Li, "Parallel driving: A novel paradigm of cloud-based unmanned driving technologies," in Proc. 28th IEEE Intelligent Vehicles Symp., Los Angeles, CA, 2017.

Corresponding Author: Fei-Yue Wang

Mailing Address: Room 1106, Intelligent Building, No. 95 Zhongguancun East Road, Haidian District, Beijing, China, 100190

Phone: 86-10-82544521

Fax: 86-10-82544521

Email: feiyue.wang@ia.ac.cn 